\title{
Modeling for Understanding v. Modeling for Numbers
}

\author{
(In Press at Ecosystems)
}

\author{
Edward B. Rastetter \\ The Ecosystems Center, Marine Biological Laboratory, Woods Hole, MA 02543
}

\begin{abstract}
I draw a distinction between Modeling for Numbers, which aims to address how much, when, and where questions, and Modeling for Understanding, which aims to address how and why questions. For-numbers models are often empirical, which can be more accurate than their mechanistic analogues as long as they are well calibrated and predictions are made within the domain of the calibration data. To extrapolate beyond the domain of available system-level data, for-numbers models should be mechanistic, relying on the ability to calibrate to the system components even if it is not possible to calibrate to the system itself. However, development of a mechanistic model that is reliable depends on an adequate understanding of the system. This understanding is best advanced using a for-understanding modeling approach. To address how and why questions, for-understanding models have to be mechanistic. The best of these forunderstanding models are focused on specific questions, stripped of extraneous detail, and elegantly simple. Once the mechanisms are well understood, one can then decide if the benefits of incorporating the mechanism in a for-numbers model is worth the added complexity and the uncertainty associated with estimating the additional model parameters.
\end{abstract}

Introduction. I draw a distinction between two types of modeling that actually represent extremes on a continuum. The first I call Modeling for Numbers. The questions addressed using these models can be summarized as: How much, where, and when? For example, how much carbon will be sequestered or released, by which parts of the biosphere, on what time course over the next 100 years (e.g., Cramer and others 2001)? The use of these models is clearly important; they address pressing environmental issues and attract a large amount of research money and effort. The second type of modeling I call Modeling for Understanding. The questions addressed with these models can be summarized as: How and why? For example, why can there be only one species per limiting factor (Levin 1970)? These for-understanding questions are more qualitative than the for-numbers questions. The emphasis of modeling for understanding is to understand underlying mechanisms, often by stripping away extraneous detail and thereby sacrificing quantitative accuracy. Modeling for understanding is at least as important as modeling for numbers (Ågren and Bosatta 1990), although the application to pressing ecological issues might be less direct.

Modeling for Numbers. There is no inherent reason why a for-numbers model has to be mechanistic. Answers to how much, where, and when can frequently be found based on past experience using purely empirical or statistical models. Such models have been used for thousands of years, for example, to know when to sow crops (e.g., after the Nile flood; Janick 2002). Modern science relies on non-mechanistic models in many ways. For example, to assess the medical risk of smoking, LaCroix and others (1991) followed 11,000 individuals, 65 years of age or older, for five years to quantify the relationship between mortality rates and smoking 
Table 1. Relative mortality rates in relation to smoking for men and women over 65. Numbers indicate the factor (and $95 \%$ confidence limit) by which mortality rates increase relative to individuals of the same sex that have never smoked. Source: LaCroix and others (1991).

\begin{tabular}{|c|c|c|}
\hline & Men & Women \\
\hline Current smoker & $2.1(1.7-2.7)$ & $1.8(1.4-2.4)$ \\
\hline Former smoker & $1.5(1.2-1.9)$ & $1.1(0.8-1.5)$ \\
\hline
\end{tabular}

(Table 1). The resulting tabular model is purely correlative and therefore cannot address the how and why connecting smoking to mortality, but it has diagnostic and predictive value. The push for "big data" approaches in Ecology hopes to capitalize on analogous analyses of large ecological data bases (e.g., Hampton and others 2013).

Empirical models are common in ecology. For example, biomass allometric equations (Yanai and others 2010), stand self-thinning relationships (Vanclay and Sands 2009), and degree-day sum phenology models (Richardson and others 2006) are all empirical models. Although various mechanisms might be hypothesized based on an examination with these models (e.g., West and Brown 2005), the models themselves have no underlying mechanism and therefore describe, rather than explain, the relationship.

Empirical models like the ones listed above have obvious value. I would further argue that in terms of producing quantitative predictions, empirical models in Biology are often, perhaps usually, more accurate than mechanistic models. For example, I cannot conceive of a mechanistic model doing as well predicting increased mortality rates with smoking as the LaCroix and others (1991) tabular model (Table 1); there is simply too much uncertainty associated with any hypothesized causal mechanism. Even if the underlying mechanism is well understood, error in estimating the parameters needed to implement a mechanistic model adds uncertainty that might overwhelm any benefit of a mechanistic approach (O'Neill 1973). I think that most empirical models are more accurate than their mechanistic analogues, with two caveats: (1) that there are enough data available to adequately calibrate the empirical model and (2) that the predictions are interpolated within the domain of the data used to calibrate the empirical model.

The weakness of empirical models is in extrapolation. Outside the domain of the calibration data there is just no way to know how well the empirical model will work, and in many cases the extrapolation is known to be poor (e.g., Richardson and others 2006). I suspect that most empirically based ecological models will not do well if extrapolated to the warmer, high- $\mathrm{CO}_{2}$ conditions of the future; the new conditions will change productivity, allometry, competition, phenology, and many other ecosystem characteristics and thereby alter the relationships underlying these models.

Of course, many of the most pressing environmental issues involve extrapolation into conditions for which there is little or no data (e.g., under future $\mathrm{CO}_{2}$ concentrations). Because of the long response times of most ecosystems, experimental approaches cannot generate the data needed to develop empirical models quickly enough to be of practical use. The only alternative is to use mechanistic models.

But why should mechanistic models be better for extrapolation than empirical models? The 
main reason is that it is often possible to empirically constrain mathematical representations of the components of a system even when it is not possible to similarly constrain an empirical representation of the whole system. Mechanistic models take advantage of the hierarchical structure of ecosystems (O'Neill and others 1986) and tie system-level behaviors to the characteristics of and interactions among the components of that system (Rastetter and Vallino 2015). Because of this hierarchical structure, the system components have to be smaller and respond more quickly than the system itself (O'Neill and others 1986), which makes them more tractable for experimental and observational study than the whole system. For example, the long-term, whole-system question to be addressed might be: Will forests sequester carbon over the next 200 years of elevated $\mathrm{CO}_{2}$ and warming? To address this question empirically at the ecosystem scale would require replicated experiments on whole forests that last 200 years. With that data one might then derive empirical relationships between initial stand biomass and soil properties and the magnitude of carbon sequestration or loss. However, such an approach is not of much use for predicting those responses for the next 200 years because the experiment takes too long. The mechanistic alternative is to instead conduct short-term experiments ( $<10$ years) on individual trees of different ages and different species, and on soils with different characteristics and then piece that information and any other available information together in a mechanistic model of the ecosystem to try to predict the long-term, whole-system rate of carbon sequestration.

This mechanistic approach also has its caveats (Rastetter 1996). Although short-term experiments can constrain representations of system components, they do not yield information about feedbacks acting at a system level when those components are linked together. Thus there is no way to know if the slow-responding system feedbacks that might dominate long-term responses are adequately represented in the model. The model might therefore be corroborated with existing short-term data even though it is inadequate for making long-term projections. Conversely, the system-level predictions of the model might be falsified with short-term, highfrequency data even though the long-term, slow-responding feedbacks that dominate long-term responses, and will eventually override the short-term, high-frequency responses, are in fact adequately represented in the model. Confidence in such models should therefore be taken with caution (Stroeve and others 2007), but should build slowly over time with the iterative process of model development and testing and the accumulation of many independent sources of corroborating evidence.

A key objective of modeling for numbers is often prediction accuracy. O'Neill (1973) postulated a tradeoff between model complexity and errors associated with estimating the parameters needed to represent that complexity in the model. As more process detail is incorporated into the model, prediction of system-level dynamics should improve because more of the processes determining those dynamics are included in the model. However, the added model complexity comes at the price of having to estimate more parameters. Error in estimating those parameters will propagate through the model and, as more parameters are added, prediction accuracy will deteriorate. Thus as the model becomes more complex there should be a tradeoff between errors associated with lack of mechanistic detail in a too-simple representation of the system (systematic error) versus cumulative errors associated with estimation of more and more parameters to account for those mechanistic details (estimation error). This tradeoff results in an optimum model complexity where overall prediction error is minimized.

The O'Neill (1973) analysis, however, presupposes that the underlying structure of the 
system being modeled is actually understood. Only if that structure is understood will added model complexity be guaranteed to reduce systematic error. If it is not understood, then the added complexity might have no relation to the real mechanism and systematic error could actually increase. Thus, there is at least one more axis to be considered in the O'Neill (1973) analysis, an axis reflecting how well the system is understood.

Modeling for Understanding. My ontological perspective is strictly reductionist; in principle, all properties of a system can be explained, and therefore understood, based on the properties of its component parts and their interactions. However, what seems straightforward in principle is often intractable in practice. The problem is the daunting complexity of biological systems. Bedau (2013) argues that some systems have interactions that are "too complex to predict exactly in practice, except by crawling the causal web." In this view, emergent system properties can be fully explained in terms of the properties of its component parts and their interactions, but that explanation might be "incompressible" in the sense that the system properties can only be replicated by simulation of the full complexity of the system (Bedau 2013).

The issue of incompressibility is hugely problematic. Taken to its extreme, it implies that a system can only be understood from the perspective of a model that is at least as complex as the system itself. What possible use could such a model be, other than to demonstrate that you have "crawled the causal web" correctly? Certainly the heuristic value of such a model would be very limited. Indeed, the formulations of many for-understanding models in ecology are selected explicitly for ease of analytical or graphical analysis, that is, for compressibility (e.g., Lotka 1925, Volterra 1926, MacArthur and Levins 1964, Tilman 1980). Achieving this compressibility requires a high degree of abstraction, a focus on a specific subset of system properties, and the sacrifice of quantitative accuracy. In exchange, there can be substantial heuristic return.

Unlike for-numbers models, which at least have accurate quantitative prediction as a common goal, it is difficult to generalize about for-understanding models except to say that they have to be mechanistic. Otherwise, how could they address how and why questions? However, a mechanistic model does not require inclusion of every process or mechanism ever described for the system. As I imply above, such an approach is counterproductive; it degrades the heuristic value of the model and therefore impedes understanding rather than enhances it. The key modeling step, and often the most difficult aspect of modeling for understanding, is identifying only those components and processes absolutely needed to address the question being asked.

The typical for-understanding model has three elements: (1) a characterization of the potential behaviors of each of the relevant system components, (2) a characterization of the interactions among these system components, and (3) a set of boundary conditions that specify, for example, the initial properties of the system components and the influence of any factors outside the system on the components of the system. The very best for-understanding models are elegantly simple.

A classic example of an elegant for-understanding model is the Lotka-Volterra model of the interactions among competing species (Lotka 1925, Volterra 1926):

$$
\frac{d N_{i}}{d t}=r_{i} N_{i} \frac{K_{i}-\sum_{j=1}^{n} \alpha_{j i} N_{j}}{K_{i}}
$$


where $N_{i}$ is the number of individuals of species $i, \boldsymbol{r}_{i}$ is the intrinsic growth parameter for species $\boldsymbol{i}, \boldsymbol{K}_{\boldsymbol{i}}$ is the number of individuals of species $\boldsymbol{i}$ that the environment is able to support in the absence of competition (carrying capacity), $\alpha_{j i}$ is the number of individuals of species $i$ that are displaced from the carrying capacity by one individual of species $\boldsymbol{j}, \boldsymbol{n}$ is the number of competing species, and $\boldsymbol{t}$ is time. The components of the system are the environment, characterized by the carrying capacities for each of the $\boldsymbol{n}$ species $\left(\boldsymbol{K}_{\boldsymbol{i}}\right)$, and the $\boldsymbol{n}$ species of competing populations, characterized by their intrinsic rates of growth $\left(\boldsymbol{r}_{\boldsymbol{i}} \boldsymbol{N}_{\boldsymbol{i}}\right)$. The environment interacts with each of the species through a density-dependent feedback that slows the rate of population growth as the population size approaches the environment's carrying capacity for that species $\left(\left[\boldsymbol{K}_{i}-\boldsymbol{N}_{i}\right] / \boldsymbol{K}_{i}\right.$; here I have assumed $\alpha_{i i}=1$; Fig. $1)$. Each species $\boldsymbol{j}$ interacts with the other species $i$ by reducing the carrying capacity of the environment for species $i$ in proportion to the abundance of species $j\left(\alpha_{j i} N_{j}\right)$. The only boundary conditions needed are the initial sizes of each of the $\boldsymbol{n}$ populations.

The Lotka-Volterra model spawned lots of research up through the early 1980s (e.g., Gause 1934, MacArthur and Wilson 1967, Parry 1981). This research sought to examine the nature of competition, the structuring of communities, and the struggle for existence. The model is still used today, but mostly as a component within larger models (e.g., Pao 2015). However, perhaps the most important legacy of this or any for-understanding model is that through its limitations it inspires a new generation of models.

The most influential of these nextgeneration models is one developed by MacArthur and Levins (1964) and further developed and applied especially by Tilman (1977, 1980):

$$
\frac{d B_{i}}{d t}=g_{i} B_{i} \min _{j=1}^{p}\left[\frac{R_{j}}{k_{i j}+R_{j}}\right]-m_{i} B_{i}
$$

$$
\frac{d \boldsymbol{R}_{j}}{d t}=S_{j}-\sum_{i=1}^{n}\left(q_{i j} g_{i} B_{i} \min _{k=1}^{p}\left[\frac{\boldsymbol{R}_{k}}{k_{i k}+\boldsymbol{R}_{k}}\right]\right)
$$

where $\boldsymbol{B}_{\boldsymbol{i}}$ is the biomass of species $\boldsymbol{i}, \boldsymbol{g}_{\boldsymbol{i}}$ and $\boldsymbol{m}_{\boldsymbol{i}}$ are the growth and turnover parameters for

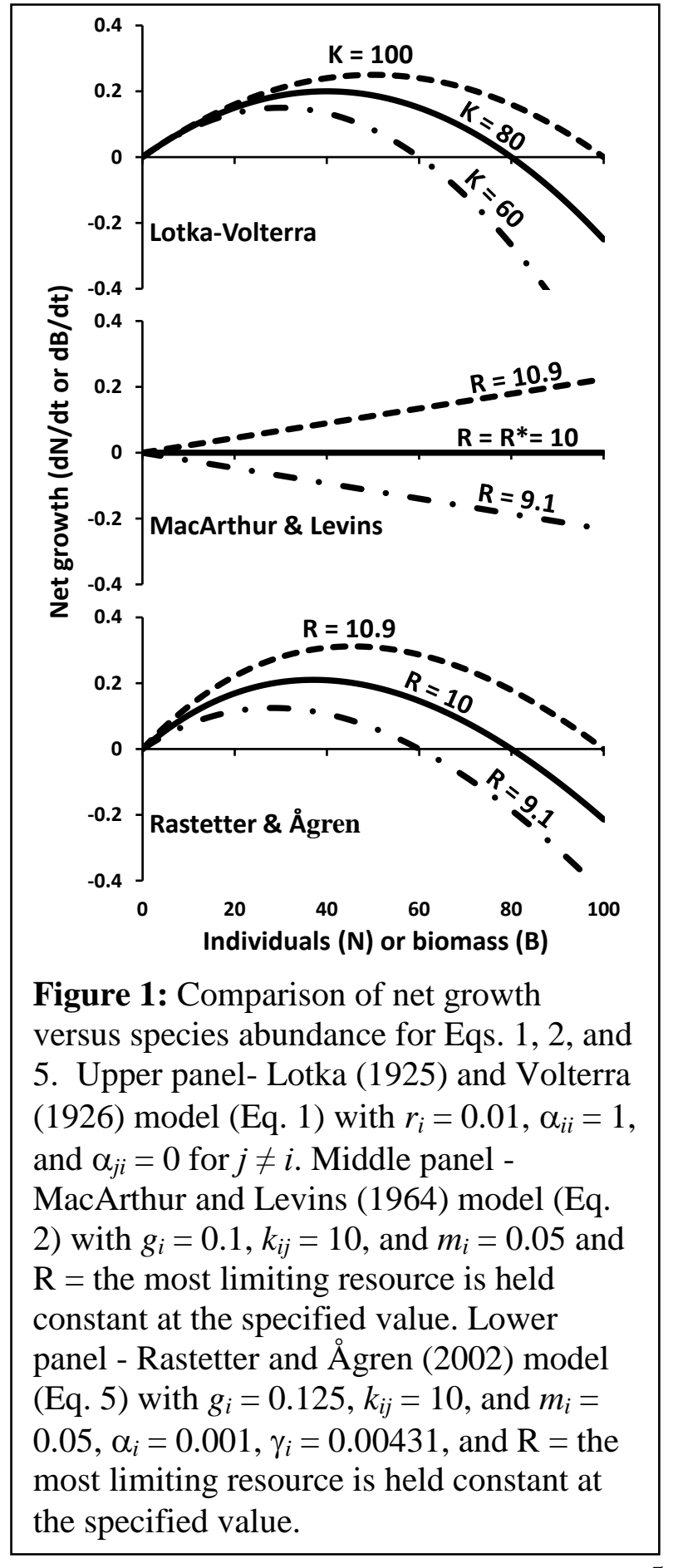


species $\boldsymbol{i}, \boldsymbol{R}_{\boldsymbol{j}}$ is the abundance of resource $\boldsymbol{j}, \boldsymbol{k}_{\boldsymbol{i} \boldsymbol{j}}$ is the half-saturation constant for uptake of resource $\boldsymbol{j}$ by species $\boldsymbol{i}, \boldsymbol{S}_{\boldsymbol{j}}$ is the net supply rate of resource $\boldsymbol{j}$ to the environment, $\boldsymbol{q}_{i j}$ is the amount of resource $\boldsymbol{j}$ needed to produce one unit of biomass for species $\boldsymbol{i}, \boldsymbol{n}$ is the number of species, $\boldsymbol{p}$ is the number of resources, and $\boldsymbol{t}$ is time. This model was developed to provide a more explicit representation of the mechanisms controlling the dynamics of the environmental resources (Eq. 3) than the earlier Lotka-Volterra model (Tilman 1987). It provided a much deeper understanding of the nature of resource limitation and competition than did a general decrease in the carrying capacity of the environment for species $\boldsymbol{i}$ by species $\boldsymbol{j}$. In addition it provided a quantitative interpretation for Gause's (1934) competitive exclusion principle $(\boldsymbol{n} \leq \boldsymbol{p})$. From Eq. 2 , at steady state in the absence of competition, species $i$ will draw down the abundance of its most-limiting resource $(\boldsymbol{j})$ to a value $\left(\boldsymbol{R}_{i j}^{*}\right)$ just able to sustain the population:

$$
\boldsymbol{R}_{i j}^{*}=\frac{\boldsymbol{k}_{i j} \boldsymbol{m}_{\boldsymbol{i}}}{\boldsymbol{g}_{i}-\boldsymbol{m}_{\boldsymbol{i}}}
$$

If any other species $\boldsymbol{k}$ is introduced into the environment and is able to draw down resource $\boldsymbol{j}$ below this level $\left(\boldsymbol{R}_{k j}^{*}<\boldsymbol{R}_{i j}^{*}\right)$ then species $\boldsymbol{i}$ will go extinct (Fig. 1). Thus, the environment can support at most $\boldsymbol{p}$ species, each with a different most-limiting resource and none able to draw down other resources below the $\boldsymbol{R}_{i j}^{*}$ of the other species.

A limitation of the MacArthur and Levins (1964) model is that it provides no resolution to Hutchinson's (1961) paradox of the plankton; in the real world, the number of coexisting species in a plankton community (and many other communities) appears to exceed the number of potentially limiting resources. Rastetter and Ågren (2002), in response to this limitation, showed that any number of species can coexist even on only one limiting resource by replacing the growth term in Eq. 2 with one that is concave downward with respect to biomass rather than proportional to biomass (Fig. 1; making turnover concave upward works equally well):

$$
\begin{aligned}
& \frac{d \boldsymbol{B}_{i}}{\boldsymbol{d} t}=\boldsymbol{g}_{i} \boldsymbol{B}_{i} \frac{\alpha_{i} \boldsymbol{B}_{i}+1}{\gamma_{i} \boldsymbol{B}_{i}+1} \min _{j=1}^{p}\left[\frac{\boldsymbol{R}_{j}}{\boldsymbol{k}_{i j}+\boldsymbol{R}_{j}}\right]-\boldsymbol{m}_{i} \boldsymbol{B}_{i} \\
& \frac{\boldsymbol{d} \boldsymbol{R}_{j}}{d t}=S_{j}-\sum_{i=1}^{n}\left(\boldsymbol{q}_{i j} \boldsymbol{g}_{i} \boldsymbol{B}_{i} \frac{\alpha_{i} \boldsymbol{B}_{i}+1}{\gamma_{i} \boldsymbol{B}_{i}+1} \min _{k=1}^{p}\left[\frac{\boldsymbol{R}_{k}}{\boldsymbol{k}_{i k}+\boldsymbol{R}_{k}}\right]\right)
\end{aligned}
$$

where $\alpha_{i}<\gamma_{i}$ are parameters that make the growth term in Eq. 5 monotonically increasing and concave downward as biomass increases. Rastetter and Ågren (2002) argue that this formulation is more realistic than Eq. 2 because the surface area through which uptake occurs increases proportionally more slowly than total biomass. For example, once a vegetation canopy closes, there is little further increase in leaf area even though the total biomass continues to increase.

In addition to allowing the coexistence of any number of species regardless of the number of potentially limiting resources, this new formulation also made several other predictions: (1) the number of coexisting species increases with the net supply rate of the resource $\left(S_{j}\right),(2)$ to displace an existing species, a new species must decrease the concentration of the limiting resource by a finite, rather that infinitesimal amount, (3) the magnitude of the decrease in the limiting resource required to displace a resident species increases as the net resource supply rate $\left(S_{j}\right)$ increases, and (4) as the net resource supply rate (and hence community productivity) increases, the Shannon-Wiener diversity first increases as the number of coexisting species 
increases, then decreases as the evenness in relative abundance of those species decreases.

Each step in this sequence of models from Lotka (1925) and Volterra (1926) to MacArthur and Levins (1964), to Rastetter and Ågren (2002) was made because of a perceived limitation in the previous model. However, the way that the limitations of for-understanding models are assessed seems to me to be qualitatively different from the way that limitations of for-numbers models are assessed. For-numbers models are assessed based on goodness of fit, precision, and accuracy of prediction. With such simple elegance, stripped of extraneous detail, testing of a forunderstanding model is more nuanced. How does one test an abstraction? Generally these models are tested in very controlled environments where extraneous factors can be minimized (e.g., chemostats, pot studies; Gause 1934, Ayala and others 1973, Tilman 1977). However, "in the field ... additional complexities are likely to occur [that] do not exist in the laboratory. Experimentally tested models, however, [might] help in the understanding of natural processes" (Ayala and others 1973). Thus, "the more general models of theoretical biology are used to deduce the form of possible solutions, rather than to predict future states of the system" (Wangersky 1978).

The importance of for-understanding models is much more qualitative and much deeper than simply to predict how much, when and where. Because the extraneous detail has been stripped from these models, it is easy to impose conditions where these models fail. However, is such a test relevant? At some level, "all models are wrong but some are [nevertheless] useful" (Box 1979). In my mind, the real value of these models is heuristic (Oreskes and others 1994). Each step in the progression along the series of models presented above represents a whole new way of thinking about the problem. Thus, the progression seems to me to be more analogous to Kuhn's (1996) paradigm shifts than to the incremental corrective steps, driven by Popper's (1968) falsification or Platt's (1964) strong inference, expected in the development of a fornumbers model.

Conclusions. For-understanding models are well developed in community and evolutionary ecology and provide a strong theoretical foundation for the science (e.g., Moore and Ruiter. 2012, or scan the titles of any volume of Theoretical Ecology). For-understanding models are less well developed in ecosystem biogeochemistry (but see Ågren and Bosatta 1996), arguably resulting in a less well established theoretical foundation (see Menge and others 2008 and Pastor 2016 for approaches that merge evolutionary ecology and biogeochemistry). Conversely, fornumbers models have made enormous strides in biogeochemistry, especially in relation to global-carbon budgets (e.g., Thornton and others 2009). The interplay between forunderstanding and for-numbers model is vital. Global carbon models are beginning to incorporate feedbacks associated with nutrient limitation and cycling; for-understanding modeling at the ecosystem scale to set a firm scientific foundation for carbon-nutrient interactions should help in that development. The interactions between community and biogeochemical processes are not well understood or incorporated in for-numbers models; again a scientific foundation based on for-understanding models is needed to resolve these issues and to identify how to best incorporate them in larger for-numbers models.

"Observation and theory get on best when they are mixed together, both helping one another in the pursuit of truth" (Eddington 1935). Students should therefore develop a broad enough understanding of theoretical modeling to be able to communicate effectively with modelers even if they do not model themselves. Modeling is a vital part of any science. In Ecology, it provides 
a way to synthesize knowledge across a diverse array of sub-disciplines and to extrapolate that knowledge to make predictions. However, the ability to predict is not the same as understanding. Understanding requires the theoretical foundation provided by for-understanding models. In general, more theoretical work characterized by modeling for understanding is needed in ecology, especially in biogeochemistry. First we need to understand our planet; then we might be able to predict the consequences of what we are doing to it and devise ways of avoiding the worst of those consequences.

Acknowledgements. This work has been supported in part by NSF grants 0949420 , 1026843, 1065587, 1107707, and 1503781. I also thank Gus Shaver, Göran Ågren, Bonnie Kwiatkowski, and Joe Vallino for many years of batting around these ideas.

\section{Citations}

Ågren, G, and E Bosatta. 1990. Theory and model or art and technology in ecology. Ecol. Model. 50: 213-220.

Ågren, G, and E Bosatta. 1996. Theoretical Ecosystems Ecology; Understanding Element Cycles. Cambridge University Press, Cambridge, UK.

Ayala, FJ, ME Gilpin, and JG Ehrenfeld. 1973. Competition between species: Theoretical models and experimental tests. Theor. Popul. Biol. 4:331-356.

Bedau, MA. 2013. Weak emergence drives the science, epistemology, and metaphysics of synthetic biology. Biological Theory 8(4):334-345.

Box, GEP. 1979. Robustness in the Strategy of Scientific Model Building. Pages 201-236 in Launer, R. L. and Wilkinson, G. N. (eds.) Robustness in Statistics. Academic Press, New York, NY.

Cramer, W, A Bondeau, FI Woodward, IC Prentice, RA Betts, V Brovkin, PM COx, V Fisher, JA Foley, AD Friend, C Kucharik, MR Lomas, N Ramankutty, S Sitch, B Smith, A White, and C Young-Molling. 2001. Global response of terrestrial ecosystems structure and function to $\mathrm{CO} 2$ and climate change: Results from six dynamic global vegetation models. Glob. Chang. Biol. 7:357-373.

Eddington, A. 1935. New Pathways in Science. MacMillan Co. New York 211pp.

Gause GF. 1934. The struggle for existence. Williams and Wilkins, Baltimore, MD.

Hampton, SE, CA Strasser, JJ Tewksbury, WK Gram, AE Budden, AL Batcheller, CS Duke, and JH Porter. 2013. Big data and the future of ecology. Front. Ecol. Environ. 11: 156-162.

Hutchinson, GE. 1961. The paradox of the plankton. Am. Nat. 95:137-145.

Janick, J. 2002. Ancient Egyptian agriculture and the origins of horticulture. Acta Hortic. 582: 23-39.

Kuhn, TS. 1996. The Structure of Scientific Revolutions, 3rd Ed. U. Chicago Press, Chicago, IL.

LaCroix, AZ, J Lang, P Scherr, RB Wallace, J Cornoni-Huntley, L Berkman, JD Curb, D Evans, and CH Hennekens. 1991. Smoking and the mortality among older men and women in three communities. N. Engl. J. Med. 324:1619-1625.

Levin, S. 1970. Community equilibria and stability, and an extension of the competitive exclusion principle. Am. Nat. 104: 413-423.

Lotka, AJ. 1925. Elements of Physical Biology. Williams and Wilkins, Baltimore, MD.

MacArthur, R, and R Levins. 1964. Competition, habitat selection, and character displacement in 
a patchy environment. Proc. Natl. Acad. Sci. USA 51:1207-1210.

MacArthur, RH, and EO Wilson. 1967. The Theory of Island Biogeography. Princeton

University Press. Princeton, NJ.

Menge, DNL, SA Levin, and LO Hedin. 2008. Evolutionary tradeoffs can select against nitrogen fixation and thereby maintain nitrogen limitation. PNAS 105: 1573-1578.

Moore, J.C., and P.C. de Ruiter. 2012. Energetic Food Webs: An Analysis of Real and Model Ecosystems. Oxford University Press, Oxford, UK.

O'Neill, RV. 1973. Error analysis of ecological models. pp 898-908 in DJ Nelson (ed) Radionuclides in Ecosystems. CONF-710501. Nat. Tech. Inform. Service, Springfield, VA.

O’Neill, RV, DL DeAngelis, JB Waide, and TFH Allen. 1986. A Hierarchical Concept of Ecosystems. Princeton University Press, Princeton, NJ.

Oreskes, N, K Shrader-Frechette, and K Belitz. 1994. Verification, validation, and confirmation of numerical models in the earth sciences. Science 263: 641-646.

Pao, CV. 2015. Dynamics of Lotka-Volterra cooporation systems governed by degenerate quasilinear reaction-diffusion equations. Nonlinear Anal-Real. 23:47-60.

Parry, GD. 1981. The meanings of r- and K-selection. Oecologia 48:260-264.

Pastor, J. 2016. Ecosystems ecology and evolutionary biology, a new frontier. Ecosystems this volume.

Platt, J.R. 1964. Strong inference. Science 146:347-353.

Popper, K. R. 1968. The Logic of Scientific Discovery. Harper and Row, New York, NY.

Rastetter, EB. 1996. Validating models of ecosystem response to global change. BioScience 46(3):190-198.

Rastetter, EB, and GI Ågren. 2002. Changes in Individual Allometry Can Lead to Species Coexistence without Niche Separation. Ecosystems 5: 789-801.

Rastetter, EB, and JJ Vallino. 2015. Ecosystem's 80th and the Reemergence of Emergence. Ecosystems 18:735-739.

Richardson, AD, AS Bailey, EG Denny, CW Martin, and J O'Keefe. 2006. Phenology of a northern hardwood forest canopy. Global Change Biol. 12:1174-1188.

Stroeve, J, MM Holland, W Meier, T Scambos, and M Serreze. 2007. Arctic sea ice decline, Faster than forecast. Geophys. Res. Lett. 34 L09501, doi: 10.1029/2007GL029703

Thornton, PE, SC Doney, K Lindsay, JK Moore, N Mahowald, JT Randerson, I Fung, J-F Lamarque, JJ Feddema, and Y-H Lee. 2009. Carbon-nitrogen interactions regulate climatecarbon cycle feedbacks: results from an atmosphere-ocean general circulation model. Biogeosciences 6:2099-2120.

Tilman, D, 1977. Resource competition between plankton algae: an experimental and theoretical approach. Ecology 58:338-348.

Tilman, D. 1980. Resources: a graphical-mechanistic approach to competition and predation. Am. Nat. 116:362-93.

Tilman, D. 1987. The importance of the mechanisms of interspecific competition. Am. Nat. 129: 769-774.

Vanclay, JK, and PJ Sands. 2009. Calibrating the self-thinning frontier. For. Ecol. Manage. 259:81-85.

Volterra, V. 1926. Fluctuations in the abundance of a species considered mathematically. Nature 118:558-560.

Wangersky, PJ. 1978. Lotka-Volterra population models. Ann. Rev. Ecol. Syst. 9:189-218. 
West, GB, and JH Brown. 2005. The origin of allometric scaling laws in biology from genomes to ecosystems: Towards a quantitative unifying theory of biological structure and organization. J. Exp. Biol. 2008:1575-1592.

Yanai, R. D., J. J. Battles, A. D. Richardson, C. A. Blodgett, D. M. Wood, and E. B. Rastetter. 2010. Estimating uncertainty in ecosystem budget calculations. Ecosystems 11:239-248. 